\title{
FACTORES PSICOSOCIALES RELACIONADOS CON EL AUTOCUIDADO EN LA PREVENCIÓN, TRATAMIENTO Y POSTRATAMIENTO DEL CÁNCER DE MAMA
}

\section{PSYCHOSOCIAL FACTORS ASSOCIATED WITH SETF-CARE IN THE PREVENTION, TREATMENT AND AFTERCARE OF BREAST CANCER}

\author{
Elisa Kern de Castro' y Lourdes Moro² \\ 1 Universidade do Vale do Rio dos Sinos, Brasil. \\ ${ }^{2}$ Universidad de Salamanca, Salamanca, España.
}

Resumen

En el presente artículo se discute acerca de los factores psicosociales asociados al autocuidado en el cáncer de mama durante la prevención, el tratamiento y el postratamiento. El autocuidado se refiere a un conjunto de actividades que la persona realiza de manera consciente para mantener su vida, su salud y su bienestar, y es necesario que sea incentivado durante todas las etapas de la enfermedad. Se presentan distintos modelos teóricos - Modelo de Creencias de la Salud, Teoría de la Acción Razonada y Modelo de Autorregulación en Salud - que intentan entender cómo y porqué las personas se cuidan en situaciones de enfermedad crónica y su aplicación en el cáncer de mama. Por último, se reflexiona sobre la importancia del estado de ánimo de la mujer afectada, de la familia y de las intervenciones psicológicas para fomentar el autocuidado en las distintas etapas de la enfermedad.

Palabras clave: Cáncer de mama, autocuidado, autogestión, psicooncología.

\section{Abstract}

This paper discusses psychosocial factors which influence self-care in breast cancer patients during prevention, treatment and aftertreatment phases. Self-care is defined as the set of activities that a patient consciously develops to look after her life, health and well-being, an it must be supported all over the different stages of the disease. Three different theoretical models are presented - Health Beliefs Model, Theory of Reasoned Action and Self-regulation Model - that try to understand how and why people practice self-care in chronic conditions and in breast cancer. Finally, the paper reflects upon the importance of women's strength of spirit, of family relations and psychological interventions to improve self-care in the different phases of the disease.

Key words: Breast cancer, self-care, selfmanagement, psycho-oncology.

\section{Correspondencia:}




\section{INTRODUCCIÓN}

El cáncer de mama es el tipo de tumor maligno más frecuente entre las mujeres de todo el mundo, que supone el $16 \%$ de todos los tipos de cáncer en la mujer ${ }^{(1)}$. La Asociación Española Contra el Cáncer $(\mathrm{AECC})^{(2)}$ señala que en España se diagnostican cada año unos 22.000 casos de cáncer de mama, lo que representa el 30\% de todos los tumores en el sexo femenino. La importancia de las cifras deriva en la especial consideración que desde la investigación y la clínica se tiene con respecto al cáncer de mama. Los hospitales generales de España cuentan con profesionales formados específicamente en cáncer de mama que se agrupan en unidades especializadas para el tratamiento y control de este tumor. Estas unidades cuentan entre sus profesionales no sólo con personal sanitario sino que paulatinamente los equipos amplían su composición, permitiendo que otros profesionales como los psicólogos, trabajen también en el control de la enfermedad buscando una mejora de la calidad de vida de las mujeres afectadas. Si bien la mayor parte de las intervenciones desde todos los ámbitos, se concentran durante el tiempo que las enfermas están en tratamiento, cada vez más se constata la importancia de trabajar el tiempo previo al diagnóstico y el tiempo posterior a la remisión de la enfermedad. En esta dirección nuestro trabajo es una reflexión sobre los aspectos psicosociales vinculados con el autocuidado y que las mujeres pueden realizar en una primera etapa de prevención, durante el desarrollo de la enfemedad y una vez que están en remisión. Las mujeres deben tomar conciencia de hasta qué punto son responsables de sus problemas de salud y ser capaces de movilizar sus propios recursos y participar activamente en mantener su salud.

En el artículo comenzamos planteando los aspectos que definen el autocuida- 
do para destacar despúes los que afectan de manera más concreta en el cáncer de mama, durante la prevención, el tratamiento y una vez que la enferma pasa a ser superviviente. Terminaremos con una reflexión sobre el autocuidado en las mujeres con cáncer de mama.

\section{Consideraciones generales sobre el autocuidado}

Es difícil hallar unanimidad con respecto al concepto de autocuidado, pues nos encontramos con un término que cambia según el foco de las investigaciones. La palabra autocuidado supone responsabilidad del individuo sobre su salud y se refiere a un conjunto de actividades que la persona realiza de manera consciente para mantener su vida, su salud y su bienestar ${ }^{(3)}$. Las prácticas de autocuidado cambian dependiendo de la situación y de la cultura e incluyen la capacidad individual de uno de comportarse y elegir sobre su salud a partir de sus conocimientos, habilidades, valores, motivación, locus de control y sentido de eficacia(4). Los pre-requisitos para el autocuidado requieren acciones volcadas para la construcción de saber y prácticas que tienen el objetivo de desarrollar y mantener la vida ${ }^{(5)}$.

Una de las premisas del autocuidado es que cualquier persona consciente y en condiciones de decidir con libertad y autonomía sabe lo que debe hacer con su cuerpo, buscando su bienestar y el bienestar de otras personas ${ }^{(6)}$. El derecho al desempeño del autocuidado solo puede ser impedido en situaciones extremas, cuando el individuo no tiene claridad sobre cómo hacerlo, y pone en riesgo su propia vida o la de otra persona. Wilkinson y Whitehead ${ }^{(4)}$ refieren que el autocuidado es un continuum que va de una responsabilidad individual sobre las elecciones de los habitos de salud y riesgos, al automanejo de la enfermedad, que puede o no incluir profesionales de salud. En ese continuum, la alianza terapéutica con el equipo sanitario es importante para ayudar en el afrontamiento de situaciones dificiles relacionadas con la salud que pueden aparecer a largo plazo.

El autocuidado en el contexto de la salud y de la enfermedad crónica muestra un movimiento que se aleja del modelo médico tradicional de cura y pone a la persona en un papel activo y de responsabilidad para con su salud (7). En términos económicos, el autocuidado ha sido el puente entre la falta y la demanda en los servicios de salud, ya que el paciente autónomo sabe cuidarse más y exige menos de estos servicios ${ }^{(4)}$. Además, el autocuidado tiene relación recíproca con el conocimiento: a mayor conocimiento más prácticas de autocuidado. Las mujeres con mayor acceso a la información y mejor estatus económico tienen una conciencia de autocuidado más desarrollada. También influyen las experiencias previas como tener un familiar enfermo de cáncer ${ }^{(8,9)}$. El miedo a un diagnóstico confirmatorio inhibe las conductas de autocuidado ${ }^{(8)}$.

Con respecto a las prácticas concretas de autocuidado, la revisión de la literatura al respecto, realizada por Johnston et al. ${ }^{(7)}$ identificó algunas conductas de autocuidado como el apoyo social, tomar la medicación y la búsqueda de información. Para Thum, Ceolin, Borges y Heck ${ }^{(10)}$, el autocuidado también afecta al contexto familiar y a la socialización de hábitos y creencias a través de las generaciones.

El concepto de autocuidado precisa que diferenciemos entre autocuidado (selfcare) y autogestión (self-management). Para Wilkinson y Whitehead ${ }^{(4)}$, ambos conceptos están relacionados con la capacidad de acción vinculada con la enfermedad y el bienestar. Estos autores refieren que la confusión se debe a que, actualmente, algunas tareas que eran realizadas tradicionalmente por el personal sanitario son practicadas ahora por el propio paciente 
(ej: monitorizar la glucosa). En este sentido, la autogestión es comprendida como parte del autocuidado ya que está relacionada con la habilidad del individuo, en conjunto con su familia, comunidad y personal sanitario, de manejar los sintomas, el tratamiento y las consecuencias físicas, psicológicas y socioculturales de la enfermedad que son inherentes al estilo de vida que requiere vivir con una enfermedad crónica. Por lo tanto, la autogestión está relacionada con la capacidad de gestionar el día a día de las personas afectadas ${ }^{(11)}$. Menéndez ${ }^{(12)}$ señala que también es necesario diferenciar entre autocuidado y autoatención, aunque sobre esta diferenciación la literatura no está muy de acuerdo. El autor considera el autocuidado como "las acciones realizadas por los individuos para prevenir el desarrollo de ciertos padecidimientos y favorecer algunos aspectos de salud positiva" (p.56). Este concepto tiene un marcado caracter individualista a diferencia del de autoatención, básicamente grupal y social y orientado hacia la salud colectiva. Plantea el autocuidado como una variante de la autoatención y señala que para los autores partidarios del concepto de autocuidado, este representaría el estilo de vida individual que permitiría reducir o eliminar las conductas de riesgo relacionadas con la salud, aunque según Menéndez esta concepcion excluye las condiciones socioeconómicas de vida, que son las que hacen posible reducir dichos riesgos.

\section{El autocuidado en el cáncer de mama}

El cáncer de mama requiere una serie de cuidados tanto de cara a su posible prevención, como durante el tratamiento y el postratamiento. El significado del autocuidado en el cáncer de mama está relacionado con diversas variables como la dimensión estética, la religión, la nutrición y su influencia en el desarrollo del cáncer y, en algunos grupos étnicos, también con la función reproductiva y el papel de la mujer en la sociedad. También es necesario conocer las representaciones sociales que tiene la mujer sobre el cáncer de mama puesto que ello influye en las prácticas de autocuidado. Según Giraldo y Arangano ${ }^{(8)}$, la representación negativa del cáncer de mama es, en parte, la responsable de que no realicen autocuidados.

Distintos modelos teóricos intentan comprender y explicar cómo y porqué las personas adoptan o no conductas de autocuidado frente a esa enfermedad. Recogemos tres de los modelos teóricos más conocidos para intentar entender la conducta de autocuidado.

a) El Modelo de Creencias de la Salud (MCS) señala que la probabilidad de que una persona adopte una conducta de salud está determinada por cuatro variables psicológicas ${ }^{(13,14)}$ :

- Vulnerabilidad percibida: la persona debe percibirse vulnerable a la enfermedad.

- Gravedad percibida: la persona tiene que considerar que las consecuencias de la enfermedad, tanto médicas como sociales, son graves.

- Beneficios percibidos; la persona necesita creer que la conducta de salud reducirá o eliminará la amenaza de la enfermedad.

- Barreras percibidas: la persona debe considerar que los inconvenientes o los aspectos negativos (físicos, psicológicos, sociales) asociados a la acción preventiva pueden ser superados.

Este modelo plantea que es preciso contar con un componente adicional en la formulación original del modelo que es la necesidad de un elemento desencadenador o «clave para la acción». Esta clave puede ser interna (por ejemplo, síntomas físicos) o externa (por ejemplo, recibir una invitación para participar en un programa de cribado). Otras variables frecuentemen- 
te añadidas al MCS son la motivación general para la salud o el valor otorgado a la salud $^{(15)}$ y las creencias sobre el locus de control de la salud ${ }^{(16,17)}$. En este caso, plantean que a mayor valor otorgado a la salud y mayor percepción de control interno sobre ésta, mayor probabilidad de llevar a cabo conductas de salud. En formulaciones posteriores del modelo ${ }^{(18,19)}$ se insiste en la necesidad de incluir explícitamente la variable autoeficacia como un elemento adicional del mismo ${ }^{(13,14)}$. Moreno y $\left.\mathrm{Gil}^{(20)}\right)$ recogen en su artículo las críticas fundamentales a este modelo y plantean que no es el más adecuado para explicar la relación entre las creencias de una persona y sus conductas de salud. Los autores plantean una explicación alternativa que atienda no solo a las conductas de salud o a las creencias que el sujeto pueda tener sino también a "las diferentes funciones que establezcan la relación entre ambas" (p.103). Así, consideramos que es un modelo apropiado para explicar las conductas de autocuidado en estas mujeres puesto que las cuatro variables señaladas están representadas: las mujeres que se sienten vulnerables a la enfermedad y que perciben como grave tener cáncer de mama, están más predispuestas a realizar conductas de autocuidado a pesar de los inconvenientes o del esfuerzo que estas conductas supongan.

b) Teoría de la Acción Razonada (TAR): plantea que el determinante más inmediato de la conducta es la intención, es decir lo que la persona piensa o se propone hacer. Estas intenciones están determinadas por dos factores, uno personal, la actitud hacia la conducta (creencia de la persona sobre las consecuencias de su conducta y el valor que le otorga a estas consecuencias) y otro social, la norma subjetiva (presión social y motivación personal para ajustarse a las expectativas) ${ }^{(21)}$. Las creencias y el valor que la persona otorga a las consecuencias que va a tener su conducta son las que determinan su acción ${ }^{(14)}$. Posteriormente Azjen ${ }^{(22)}$ añade a esta teoría un nuevo elemento: el grado en que el sujeto percibe que la conducta está bajo su propio control. El autor formula así la Teoría de la Conducta Planificada (TCP). A pesar de la semejanza con el modelo de creencias de la salud, la Teoría de la Acción Razonada incluye la importancia del factor social en las conductas de salud del individuo ${ }^{(13,23)}$. La intención personal es importante para las conductas de salud, pero hay que añadir las percepciones del individuo sobre las expectativas de las personas sobre su salud, y cómo él piensa que puede corresponder a tales expectativas ${ }^{(23)}$.

c) Modelo de autorregulación en salud: la idea principal es que la percepción que la persona tiene sobre una enfermedad guía sus acciones relacionadas con la salud y la manera como afronta el problema $^{(24-26)}$. Del mismo modo, la percepción del riesgo que un individuo puede tener acerca de una enfermedad, como el cáncer de mama, es uno de los factores que pueden influenciar el afrontamiento de esa realidad y, por lo tanto, su autocuidado $^{(27,28)}$. Aunque los mecanismos de autorregulación requeridos en ese caso aún no están bien establecidos, saber que su vida es vulnerable a cierta enfermedad puede ser un factor clave para impulsar reacciones y conductas relacionadas al 'estar en riesgo', como es el autocuidado. Así, las reacciones desencadenadas por la percepción de estar en riesgo pueden interactuar con las percepciones individuales preexistentes sobre las posibles causas de la enfermedad ${ }^{(29)}$.

La presencia de un síntoma en el cuerpo activa el proceso de autorregulación ${ }^{(27)}$. Por un lado, síntomas no familiares pueden motivar que la persona haga un autodiagnostico equivocado y no busque ayuda. Por otro lado, la presencia de un síntoma familiar puede activar la percepción de riesgo, la búsqueda de ayuda y 
el autocuidado de la paciente frente a su posible enfermedad. La historia familiar de cáncer puede también tener un impacto sobre la percepción de la enfermedad y autocuidado $^{(30)}$. Al tener la experiencia cercana de un familiar o amigo con cáncer, y dependiendo de las consecuencias que tuvo, la persona puede creer que la enfermedad es evitable o no, es tratable o no, lo que tiene relación con el autocuidado. Así, para Constanzo, Lutgendorf y Röeder(31), el modelo de autorregulación en salud ha sido útil en predecir prácticas de salud en pacientes con cáncer, y en ayudar a los enfermos a reconstruir su vida de forma sana después del final del tratamiento.

\section{El autocuidado en la prevención del cáncer de mama}

Conforme ya se ha mencionado, la percepción del grado de riesgo de tener una enfermedad grave como el cáncer de mama es uno de los factores que pueden guiar las conductas de autocuidado. Riesgo significa cualquier factor que incrementa la posibilidad de tener una enfermedad, en este caso, el cáncer de mama(2).

La $\mathrm{AECC}^{(2)}$ señala distintos factores relacionados con el riesgo de cáncer de mama: 1) factores que no se pueden modificar, como sexo, edad, raza, hereditarios, etc; 2) factores que se pueden cambiar como descendencia, factores hormonales exógenos, factores dietéticos y ejercicio físico; 3) Factores inciertos como la polución ambiental, tabaco, cafeína, ingesta prolongada de antiinflamatorios, fitoestrógenos, golpes en las mamas, aros en los sujetadores, depilación de las axilas, mamas de diferente tamaño, tamaño del pecho, etc. y 4) factores ambientales como la exposición a radiaciones ionizantes. La presencia de los oncogenes (BRCA1, BRCA2) es un factor importante para la aparición del cáncer de mama, aunque en muchos casos la enfermedad surge sin que haya riesgo genético. Además, en el 50\% de los casos la enfermedad también puede aparecer en mujeres que no tienen, al menos aparentemente, factores de riesgo para el cancer de mama ${ }^{(32)}$. Así, la escasa vinculación genética del cáncer de mama permite plantear que la vulnerabilidad del comportamiento, de la expresión de emociones y de la personalidad son posibles factores que actúan en su desarrollo, aunque esa relación no está clara ${ }^{(23,33,34)}$. Parra, García e Insuasty ${ }^{(32)}$ recogen que los estudios cualitativos sobre el significado cultural del cáncer de mama han identificado explicaciones diversas sobre el origen del mismo, como la represión de sentimientos, el estrés, la falta de cuidado de la salud y los malos hábitos alimenticios. Sin embargo, como el cáncer es una enfermedad con multiplos factores ${ }^{(1)}$, no es posible confirmar tales explicaciones.

Es importante que el riesgo percibido se utilice junto con la información sobre prevención en el cancer de mama ${ }^{(27,35)}$. Se espera que el alto riesgo percibido motive a la persona a realizar actividades de cuidado con su salud. No obstante, es sabido desde hace mucho tiempo en Psicologia de la Salud que los estímulos carcinógenos generan efectos de satisfacción a corto plazo (ej., mala alimentación, tabaco, etc.) mientras el cuidado con la salud y la prevención primaria del cáncer pueden no tener efecto a largo plazo, visto que hay personas que desarrollan la enfermedad sin tener factores de riesgo asociados ${ }^{(36)}$. Así, es importante informar a las personas sobre los posibles efectos a largo plazo de sus conductas relacionadas con la salud, pero eso no es suficiente para el cambio de comportamiento de muchas de ellas.

Con todo, no todos los individuos quieren o son competentes para tener cuidados con su salud ${ }^{(7)}$. Según la literatura, los factores de riesgo relacionados con el cáncer de mama, son 'solamente' riesgos. Para algunas personas, alimentarse bien o hacer ejercício físico, por ejemplo, son esfuerzos 
que no merecen la pena para una situación que es solo 'probable' de ocurrir. La satisfacción inmediata de hacer algo que le gusta es más fuerte que el riesgo futuro, y éste puede ser uno de los factores que dificultan el autocuidado. Según Ozanne y Esserman $^{(37)}$ las medidas preventivas en el cáncer de mama, como las modificaciones en el estilo de vida, la quimioprevención y la cirugía profiláctica, están infrautilzadas. Giraldo y Arango $^{(8)}$ señalan que a pesar de la importancia de la detección temprana, las prácticas de autocuidado como el autoexamen, el examen clínico y la mamografía no se hacen con la periodicidad necesaria.

Por otro lado, existen aspectos culturales relacionados con el rol de la mujer en la sociedad. En muchas culturas, la mujer es la que cuida de los demás y, por lo tanto, se olvida de su propio cuidado ${ }^{(38)}$. Además, pueden existir barreras psicológicas para realizar el autoexamen o pueden preferir ser atendidas por profesionales del mismo género por la vergüenza de mostrar su cuerpo a médicos hombres. De hecho, McQueen et al. ${ }^{(35)}$, en un estudio epidemiológico, concluyeron que existe diferencia en la percepción de riesgo de padecer cáncer entre hombres y mujeres. Las mujeres suelen ver la historia familiar de cáncer como un factor de riesgo más importante para su salud que los hombres.

A la hora de poder realizarse mamografías como factor de prevención, nos encontramos que en algunos países existen dificultades, tanto personales porque no acuden a las citas, como institucionales porque la sanidad pública no lo permite. Las largas listas de espera para realizar pruebas de control influyen en la motivación para el autocuidado.

Galdón, Durá, Andreu y Tuells ${ }^{(13)}$ y Andreu, Galdón y Durá(14) señalan tres tipos posibles de predictores de la asistencia a un programa de cribado mamográfico: 1) Variables sociodemográficas: la asistencia al programa se relaciona con una menor edad y un mayor nivel de estudios; 2) Conductas preventivas previas: confirmando los resultados de numerosos trabajos anteriores, señalan que el poseer una orientación preventiva hacia la salud y llevar a cabo otras conductas preventivas juega un papel importante a la hora de participar en el programa de mamografía; 3) Creencias de salud: la mayor gravedad percibida se perfila como un factor que inhibe la participación en el programa de cribado. Estos autores señalan que la creencia de controlabilidad sobre la enfermedad favorece la realización de conductas preventivas del cáncer de mama. Una mayor información respecto a las técnicas de detección temprana del cáncer de mama también favorece la participación en programas de cribado mamográfico.

También la cuestión de la asistencia a los servicios medicos y la relación medicopaciente pueden ser factores que dificultan las conductas de autocuidado. Algunas veces hay falta de interés por parte del equipo sanitario de consolidar una buena relación con las pacientes ${ }^{(38)}$.

\section{El autocuidado en la fase de tratamiento}

El principal objectivo del autocuidado en el tratamiento de una enfermedad crónica es el manejo de los síntomas, prevención de complicaciones y ajustes en los habitos de vida ${ }^{(39)}$. Las dificultades en el autocuidado durante el tratamiento, en general, ocurren en función de las fluctuaciones de los síntomas en el proceso de la enfermedad.

Las prácticas de autocuidado en pacientes enfermos pueden ser confundidas con el término adhesión al tratamiento ${ }^{(40)}$. La adhesión es definida como la conducta de la persona en relación a su enfermedad que está relacionada con la recomendación médica, y puede incluir la ingestión correcta de la medicación, dieta, cambios 
en su vida, etc. ${ }^{(41,42)}$. El autocuidado es un concepto más amplio y activo y está cercano al de empoderamiento y responsabilidad del individuo sobre su salud ${ }^{(4)}$. Por otro lado, el concepto de adhesión puede implicar mayor pasividad ya que tiene relación directa con el seguimiento de las prescripciones del equipo sanitario.

En el caso concreto del cáncer de mama las prácticas de autocuidado se relacionan directamente con los efectos secundarios que aparecen como consecuencia de los tratamientos con respecto a la imagen corporal y al estado de ánimo.

Para Ridner, Dietrich y Kidd(43), las pacientes con cáncer de mama no reciben información suficiente sobre el autocuidado que deben tener durante su tratamiento, en especial para evitar el linfedema. En su estudio realizado con 51 pacientes, los autores verificaron que las mujeres creen que las informaciones recibidas son insuficientes para evitar y cuidar el linfedema, y que usan pocos minutos al día en su autocuidado, concretamente el 33\% de las pacientes emplean menos de 15 minutos.

Loh, Tan y Xavier ${ }^{(44)}$ realizaron un estudio en el que trataron de disminuir los niveles de depresión, ansiedad y estrés en mujeres con cáncer de mama mediante una intervención de apoyo a la autogestión de conductas de salud relacionadas con el conocimiento y las habilidades necesarias para gestionar las tareas médicas, emocionales y el rol de la mujer que tiene que vivir con cáncer de mama. En este trabajo apuntaron el papel beneficioso que tiene hacer ejercicio físico durante el tratamiento para disminuir la tensión y el estrés, aunque señalan que deben realizarse más estudios al respecto para poder certificar este resultado de forma más significativa. En este sentido, Loh, Chew, Lee y Quek ${ }^{(11)}$ han desarrollado un programa de autogestión- self-management- del cáncer de mama denominado SAMA (Stay Abreast, Move Ahead) pues consideran que es fun- damental considerar el papel que tiene la propia mujer en la gestión de su salud. El objetivo general del programa es mejorar su calidad de vida proporcionando la información y las habilidades que las afectadas necesitan para poder gestionar el día a día como enfermas y posteriormente como supervivientes.

\section{El autocuidado en el postratamiento}

El Grupo Español de Pacientes con Cáncer $\left(\mathrm{GEPAC}^{(45)}\right)$ ha realizado en el año 2012 el Informe sobre las necesidades de los supervivientes de cáncer en el que apuntan que en la actualidad hay en España un millón y medio de personas que han sobrevivido al cáncer. Estas personas aunque libres de la enfermedad arrastran importantes secuelas físicas y psicosociales que es necesario conocer y para las que necesitan ayuda.

No se discutirá aquí la cuestión de lo que es superviviente del cáncer, debido a que no hay unanimidad en el concepto $y$ a que no es relevante para los objetivos de este artículo ${ }^{(46)}$. Nos interesa conocer el periodo postratamiento ya que requiere una serie de cuidados para las pacientes que incluyen no sólo las visitas y pruebas sistemáticas para verificar que siguen libres de la enfermedad, sino también cambio de conductas con el objetivo de evitar la recidiva o para manejar posibles efectos secundarios de la enfermedad y su tratamiento. Utilizamos el término postratamiento para referirnos a las prácticas de autocuidado que deben comenzar inmediatamente después de terminar los tratamientos a que haya sido sometida.

Los factores de riesgo relacionados con un impacto psicológico negativo del cáncer en los supervivientes son variados y numerosos. Entre ellos los autores destacan variables sociodemográficas, clínicas y culturales como: la edad y el momento del ciclo vital en el que se produjo el diag- 
nóstico, el tipo de cáncer, el curso de la enfermedad, los síntomas somáticos más frecuentes, el bajo funcionamiento físico, una visión pesimista del pronóstico de la enfermedad, las creencias culturales sobre el carácter amenazante del cáncer o características de personalidad. Las dificultades psicológicas son mayores cuanto más serias hayan sido estas circunstancias ${ }^{(47)}$. Harrington et al. ${ }^{(48)}$ señalan que los supervivientes de cáncer manifiestan limitaciones físicas y cognitivas, depresión y ansiedad, problemas de sueño, fatiga, dolor y disfunciones sexuales. Estos síntomas pueden experimentarlos incluso 10 años después de recibir tratamiento para el cáncer. Para paliar estos síntomas plantean que debe recurrirse a opciones farmacológicas, educativas, intervenciones psicosociales y autogestión.

El miedo a la recurrencia de la enfermedad acompaña a los supervivientes durante mucho tiempo después de que la enfermedad ha pasado ${ }^{(49)}$. Muchas pacientes tienen miedo a acudir a las consultas de revisión por recelo a las malas noticias y la posibilidad de recidiva. Además, el final de los tratamientos puede aumentar el estrés y la angustia de las enfermas ya que pierden el cuidado "seguro" que recibían hasta este momento ${ }^{(31)}$.

Constanzo, Lutgendorf y Roeder ${ }^{(31)}$ han realizado un estudio que tenía como objetivos 1) conocer en que medida los pacientes supervivientes de cáncer de mama manifiestan cambios de comportamiento 3 semanas y 3 meses después del tratamiento, y qué conductas están mas preparados para cambiar; 2) caracterizar las creencias sobre el cáncer usando el modelo de percepción de la enfermedad de Leventhal; 3 ) relacionar las creencias con los cambios de comportamiento al finalizar el tratamiento. Participaron 71 mujeres en las dos etapas del estudio, con edades entre 32 y 89 años. Los resultados mostraron que las supervivientes hacen esfuerzos para cam- biar sus conductas al final del tratamiento, como aumentar la actividad física, dejar de fumar y no beber alcohol. Las pacientes que modificaron estas conductas obtuvieron mejores resultados en las practicas de salud examinadas. Las mujeres que creían que el cáncer tiene consecuencias más graves y las que atribuyen las causas y la prevención del cáncer a conductas de salud o estrés estan más dispuestas a cambiar de actitudes, mejorar su dieta y realizar más actividad fisica en el posttratamiento. Por ello, los autores creen que el periodo postratamiento inmediato es oportuno para promover cambios en los cuidados con la salud.

Aires-González, Beato y Virizuela ${ }^{(47)}$ recogen la propuesta que la Universidad del Paciente ha hecho en un estudio titulado Después del Cáncer. En este trabajo reflejan la opinión de expertos y afectados que concluyen que es necesario, entre otros aspectos, diseñar un Plan de Seguimiento específico para supervivientes, crear Unidades de atención especializadas, facilitar la atención y el seguimiento integral del superviviente por un equipo multidisciplinar y con un oncólogo de referencia, diseñar programas de formación en estilos de vida saludables, conseguir mayor evidencia acerca del impacto psicológico en personas supervivientes a largo plazo, información tras los tratamientos, orientación en temas relacionados con la pareja, familia o reinserción laboral, contacto con otros afectados, atención al cuidador principal y programas de formación para profesionales en habilidades de comunicación.

Las campañas de educación para la salud deben insistir en la importancia de fomentar el papel activo de la mujer en el cuidado de su salud y sus repercusiones a nivel preventivo ${ }^{(13,14)}$. Debe fomentarse el autocontrol sobre la salud, es decir la creencia en que uno puede hacer algo por su salud, y disminuir la amenaza perci- 
bida de algunas enfermedades como el cáncer de mama. Las campañas en este caso deberían proporcionar información sobre las distintas estrategias de detección temprana de la enfermedad, fomentando la actitud positiva hacia las mismas y, especialmente, enfatizar los beneficos de la mamografía, aumentando la presión social percibida para Ilevarla a cabo. Según los autores estas campañas podrían incluir información sobre la incidencia del cáncer de mama y sus factores de riesgo con el objetivo de aumentar la percepción de vulnerabilidad a esta enfermedad, simpre incluyendo mensajes tranquilizadores que no aumenten la gravedad percibida de la enfermedad y el nivel general de amenaza percibida a la salud

\section{Perspectivas futuras y consideraciones finales}

Aunque el cáncer de mama sea una enfermedad que tiene tratamientos eficaces cuando es detectado temprano y los factores de riesgo son conocidos, nadie está libre de padecerlo, ni siquiera las personas que tienen mucho cuidado con su salud. A continuación proponemos una reflexión sobre las prácticas de autocuidado en el cáncer de mama que pueden ayudar al equipo asistencial a incrementar las conductas de autocuidado de estas pacientes.

Es evidente que el autocuidado en la prevención es importante aunque no sea al $100 \%$ eficaz para evitar la enfermedad. En el ámbito de la prevención es necesario, no solamente explicar cuáles son los factores de riesgo y la necesidad de realizar revisiones periodicas, sino también informar sobre el aumento de las probabilidades de tener cáncer en ausencia de autocuidado con su salud. Ofrecer datos objetivos sobre cuánto aumenta el riesgo de padecer cáncer por realizar determinados comportamientos durante un cierto tiempo, podría generar en la persona una mayor conciencia de responsabilidad para con su salud.

El autocuidado requiere intervenciones psicosociales y educativas multidisciplinares que consideren las necesidades de las personas sobre su salud y las propias soluciones que ellas proponen, partiendo de los recursos de que dispone la comunidad. Las propuestas educativas sobre el autocuidado tienen que formar parte de la educación para la salud que debe inculcarse desde los primeros años de vida. Es fundamental que en los colegios se enseñe a las niñas a conocer su cuerpo y a saber que pueden realizar distintas conductas a lo largo de su vida que pueden influir positivamente en su salud.

La familia es otro aspecto que es necesario trabajar fomentando la participación del entorno familiar en los cuidados de salud, de manera que la mujer sienta que es importante para los demás que ella se cuide, pero a la vez el entorno debe permitir y estimular a la mujer para que tenga su propia autonomía. En este ámbito es especialmente relevante el papel de la pareja, que debe involucrarse y apoyar las conductas de autocuidado de su mujer, ya que la relación sufre importantes cambios (por ejemplo, disminución de la libido, alteraciones anímicas, repercusiones en la imagen física) durante todo el proceso de la enfermedad y una vez que la paciente está curada.

Durante todas las fases de la enfermedad debe trabajarse la autoimagen y la autoestima de la mujer, el conocimiento sobre su propio cuerpo y la importancia de que sea consciente de síntomas que pueden indicar alguna enfermedad.

En lo que se refiere al autocuidado durante el tratamiento, la falta de conocimiento sobre sus efectos adversos puede generar sorpresa, sufrimiento e inseguridad y ser uno de los más importantes obstaculos para el autocuidado. El rol de enfermo que la persona asume y que muchas veces es 
reforzado por el equipo sanitario y su familia no ayuda a la paciente a tener un papel activo con su enfermedad. Es importante fomentar el autocuidado durante el tratamiento para ayudar a la mujer a tener mayor percepción de control sobre su cuerpo, su enfermedad y su vida, lo que aumenta la sensación de bienestar y su calidad de vida. Durante el tratamiento las conductas de autocuidado deben ir dirigidas a trabajar las repercusiones de los efectos secundarios relacionados con los cambios en la imagen corporal como la pérdida o ganancia de peso, la alopecia, problemas en la piel o en las uñas, que pueden motivar que la mujer no se reconozca o no se sienta la misma persona de antes. También es necesario intervenir en el estado de ánimo que se ve afectado desde que la mujer conoce el diagnóstico y se agrava con los efectos secundarios de los tratamientos. Las personas con depresión tienden a no cuidarse y es necesario que se potencie su participación en el tratamiento.

Cuando la mujer está en remisión parece el mejor momento para cambiar conductas inadecuadas. Al igual que durante el tiempo que está enferma, una vez que la enfermedad remite, las prácticas de autocuidado deben estar enfocadas a trabajar en las posibles secuelas físicas, emocionales y sociales. La mujer debe volver a su vida anterior a la enfermedad, pero siendo consciente de los cambios que ha sufrido y de las repercusiones que ello tendrá el resto de su vida.

En todas las etapas de la enfermedad es necesario el trabajo del psicooncologo que enfatize la salud y la supervivencia, valorando la victoria del tratamiento y la fuerza de la persona para vencerlo.

\section{REFERENCIAS BIBLIOGRÁFICAS}

1. World Health Organization (WHO). Breast cancer, prevention and control. [En línea] 2012 [Acceso 28/05/2012]. Disponible en: http://www.who.int/cancer/detection/ breastcancer/en/index1.html, 2012

2. Asociación Española Contra el Cáncer (AECC). Cáncer de mama [En línea] 2012 [Acceso 28/05/2012]. Disponible en: https://www.aecc.es/SobreElCancer/ CancerPorLocalizacion/CancerMama/ Paginas/cancerdemama.aspx.

3. Leopardi MT. Teorias em enfermagem: instrumentos para a prática. Florianópolis (SC): Ed. Papas, 1999.p. 241-52.

4. Wilkinson A, Whitehead L. Evolution of the concept of self-care and implications for nurses: A literature review. Int J Nurs Studies 2009,1:1143-7. Doi: 10.1016/j. ijnurstu.2008.12.011,

5. Silva IJ. Cuidado, autocuidado e cuidado de si: uma compreensão paradigmática para o cuidado de enfermagem. Rev Esc Enf USP 2009;43:697-703.

6. Lunardi VL, Lunardi Filho WD, Silveira RS, Soares NV, Lipinski JM. O cuidado de si como condição para o cuidado dos outros na prática de saúde. Rev Latino-Am Enfermagem. nov/dez 2004; 12 (6):933-9.

7. Johnston B, McGill M, Milligan S, McElroy D, Foster C, Kearney N. Self-care and end of life care in advanced cancer: A literature review. Eur J Oncol Nurs 2009;13:386-98. Doi: 10.1016/j.ejon.2009.04.003,

8. Giraldo-Mora CV, Arango ME. Representaciones sociales frente al autocuidado en la prevención del cáncer de mama. Inv Educ Enf 2009;27(2):191-200.

9. Ceballos GY, Giraldo CV. Autobarreras de las mujeres al diagnóstico y tratamiento oportuno del cáncer de mama. Aquichan 2011;11(2):140-57.

10. Thum MA, Ceolin T, Borges AM, Heck RM. Saberes relacionados ao autocuidado entre mulheres da área rural do sul do Brasil. Rev Gaúcha Enf 2001;32(3):576-82.

11. Loh SY, Chew SL, Lee SY, Quek KF. Quality of life in breast cancer survivors: 2 years post self-management intervention. Asian Pac J Cancer Prev 2011;12:1497-501.

12. Menéndez E. Intencionalidad, experiencia 
y función: la articulación de los saberes médicos. Rev Antropol Social 2005;14:3369.

13. Galdón MJ, Durá E, Andreu Y, Tuells J. Creencias de salud relacionadas con la participación en un programa de cribado mamográfico. Psicol Conduct 2000;8(2):357-73. Doi:10.5209/rev_ PSIC.2012.v9.n1.39135

14. Andreu Y, Galdón MJ, Durá E. Edad, creencias de salud y asistencia a un programa de cribado mamográfico en la Comunidad Valenciana. Rev Esp Salud Pub 2004;78(1):65-82.

15. Becker $\mathrm{MH}$. The health belief model and personal health behavior. Health Education Monogr 1974;2:324-473

16. Wallston KA, Wallston BS. Health locus of control scales. In: Lefcourt $\mathrm{H}$, editor. Research with the locus of control construct. Nueva York: Academia Press, 1981.p.189243.

17. Lau RR. Beliefs about control and health behavior. In: Gochman DS, editor. Health behavior: emerging research perspectives. Nueva York: Plenum, 1988, p.43-63.

18. Rosenstock IM, Strecher VJ, Becker $\mathrm{MH}$. Social learning theory and the health belief model. Health Educ Q 1998;15:17583.

19. Rosenstock IM. The health belief model: Explaining health behavior through expectancies. In: Glanz K, Lewis FM, Rimer BK, editors. Health behavior and health education: theory, research and practice. San Francisco: Jossey-Bass, 1990, p.39-62.

20. Moreno E, Gil J. El modelo de Creencias de Salud: revisión teórica, consideración crítica y propuesta alternativa. Hacia un análisis funcional de las creencias en salud. Int J Psych Psychol Therapy 2003;3(1):91-109.

21. Ajzen I, Fishbein M. Attitude-behavior relations: A theoretical analysis and review of empirical research. Psychol Bull 1977;84:888-918.

22. Azjen I. The theory of planned behavior.
Organ Behav Hum Dec Process 1992;50:179-211.

23. Durá E, Ibañez E. Psicología Oncológica: perspectivas futuras de investigación e intervención profesional. Psicol Saúde \& Doenças 2000;1(1):27-43.

24. Leventhal H, Nerenz DL, Steele DJ. Illness representation and coping with health threats. In: Baum A, Taylor SE, Singer JE, editors. Handbook of Psychology and Health, Volume IV: Social psychological aspects of health. Hillsdale, NJ: Erlbaum 1984. p.219-52.

25. Leventhal $H$, Benyamini $Y$, Brownlee $S$, Deifenbach $M$, Leventhal EA, PatrickMiller L, Robitaille C. Illness representations: theoretical foundations. En: Petrie KJ, Weinman JA, editors. Perceptions of health and illness. Harwood Academic Publishers, 1997. p.19-46.

26. Leventhal H, Brissette I, Leventhal EA. The common-sense model of self-regulation of health and illness. In: Cameron LD, Leventhal $H$, editors. The self-regulation of health and illness behaviour. London: Routledge, 2003. p.42-65.

27. Bish A, Ramirez A, Burgess C, Hunter M. Understanding why women delay in seeking help for breast cancer symptoms. J Psychosom Res 2005;58: 321-6.

28. Shiloh S, Drori E, Orr-Urtregger A. Being 'at risk' for developing cancer: Cognitive representations and psychological outcomes. J Behav Med 2009;32:197-208. Doi:10.1007/s10865-008-9178-z

29. Marteau TM, Weinman J. Self-regulation and the behavioral response to DNA risk information: A theoretical analysis and framework for future research. Soc Sci Med 2006; 62:1360-8. Doi: 10.1016/j. socscimed.2005.08.005,

30. Rees G, Fry A, Cull A, Sutton S. Illness perceptions and distress in women at increased risk of breast cancer. Illness perceptions and distress in women at increased risk of breast cancer. Psychol Health 2004; 19:749-65. 
31. Constanzo ES, Lutgendorf LK, Roeder S. Common-sense beliefs about câncer and health practices among women completing treatment for breast cancer. Psychooncology 2011; 20:53-61. Doi: 10.1002/pon.1536

32. Parra CE, García LV, Insuasty J. Experiencias de vida en mujeres con cáncer de mama en quimioterapia. Rev Colomb Psiq 2011;40(1):65-84.

33. Härtl K, Engel J, Herschbach P, Reinecker $\mathrm{H}$, Sommer H, Friese K. Personality traits and psychosocial stress: Quality of life over 2 years following breast cancer diagnosis and psychological impact factors. Psychooncology 2010; 19(2):1609. Doi: 10.1002/pon.1536

34. Michael IL, Carlson NE, Bowen JD, Ritenbaugh C, Chlebowski RT, Weihs KL, Ockene JK, Invited commentary: Personality as a causal factor in cancer risk and mortality - time to retire a hypothesis. Am J Epidemiol 2011;173:716. Doi:10.1093/ aje/kwq448

35. McQueen A, Vernon SW, Meissner HI, Rakowski W. Risk perceptions and worry about cancer: Does gender make a difference? J Health Communic 2008;13:5679. Doi: 10.1080/10810730701807076

36. Bayés R. Psicología Oncológica. Barcelona: Martínez Roca, 2000.p.186.

37. Ozanne EY, Esserman L. Decision making in breast cancer prevention. Psicooncología 2010;7(2-3):299-311.

38. Poblano-Verastegui O, Figueroa-Perea JM, López-Carrillo J. Condicionantes institucionales que influyen en la utilización del exámen clínico de mama. Salud pública de Mexico 2004; 46(4):294.

39. Williams B, Pace AM. Problem based learning in chronic disease management: a review of the research. Patient Educ Couns 2009;77:14-9. Doi: 10.1016/j. pec.2009.03.004,

40. Santos I, Sarat CN. Modalidades de aplicação da teoria do autocuidado de Orem em comunicações científicas de enfermagem brasileira. Rev Enferm 2008; 16(3)313-8.

41. Pierin AMG, Strelec MAAM, Mion Júnior D. O desafio do controle da hipertensão arterial e a adesão ao tratamento. In: Pierin AMG, editor. Hipertensão arterial: uma proposta para o cuidar. Barueri: Manole; 2004. cap 16. p.275-89.

42. Pinho LS, Campos AC, Fernandes AFC, Lôbo SA. Câncer de mama: da descoberta à recorrência da doença. Rev Eletron de Enferm 2007:9(1):154-65.

43. Riddner SH, Dietrich MS, Kidd, N. Breast cancer treatment-related lymphedema self-care: education, practices, symptoms, and quality of life. Supp Care Cancer 2011;19:631-7. Doi:10.1007/s00520-0100870-5

44. Loh SY, Tan FL, Xavier M. Depression, anxiety and stress in women with breast cancer: Effect of a 4-week self management intervention. Malasyan J Psych 2009;18(1):1-9.

45. Grupo Español de Pacientes con Cáncer (GEPAC). Informe sobre las necesidades del superviviente de câncer [En línea] 
